\title{
Photochemistry of Complex Ions. VII.
}

\section{trans-4-Stilbenecarboxylatopentaamminecobalt(III) Ion}

\author{
by Arthur W. Adamson, Arnd Vogler, and Ian Lantzke ${ }^{1}$ \\ Department of Chemistry, University of Southern California, Los Angeles, California 90007 \\ (Received A pril 28, 1969)
}

\begin{abstract}
The aqueous ion $\mathrm{Co}\left(\mathrm{NH}_{3}\right)_{5}(\mathrm{TSC})^{2+}$, where TSC denotes trans-4-stilbenecarboxylate, appears in most respects to be a normal member of the carboxylatopentaamminecobalt(III) family. The second ligand field band is masked, however, by a strong absorption at $320 \mathrm{m \mu}$, essentially identical with that for the free TSC molecule. The first singlet-singlet transition of the TSC moiety thus appears to function as an isolated chromophoric group of the complex. Irradiation of the $320-\mathrm{m} \mu$ band of the free TSC ligand leads to trans to cis isomerization; however, irradiation of this same band in the complex produces cobalt(II) in a quantum yield of 0.16 and both some free (unisomerized) and some oxidized TSC ligand. Other members of the carboxylatopentaamminecobalt(III) series are nearly inert to this wavelength of irradiation. Free TSC-ion shows a peak fluorescent emission at $400 \mathrm{~m} \mu$, which is partially quenched in the presence of $\mathrm{Co}\left(\mathrm{NH}_{3}\right)_{5}\left(\mathrm{H}_{2} \mathrm{O}\right)^{8+}$ and essentially completely so when the ligand is coordinated. It is concluded that an intramolecular excitation energy transfer occurs, probably to produce a charge transfer triplet excited state of the complex, which then undergoes redox decomposition. The suggested process is one of intersystem crossing from the first singlet excited state of the TSC ligand.
\end{abstract}

\section{Introduction}

The present investigation was undertaken to determine whether an intramolecular transfer of excitation energy leading to photolysis could be observed for a Werner-type complex ion. Certain cobalt(III) and other chelates have been reported to quench organic triplet excited states, ${ }^{2-4}$ but the effect could be attributed to a catalysis of the radiationless return of the donor to the ground state. Excitation energy transfer clearly occurs, however, in the case of certain rare earth chelates, for which irradiation of ligand localized absorption bands lead to emission characteristic of the rare earth ion. ${ }^{5}$ Similar evidence has been reported for solid $\left[\mathrm{Cr}(\text { urea })_{6}\right]\left[\mathrm{Cr}(\mathrm{CN})_{6}\right]$; in this case low-temperature irradiation of a cation absorption band led to emission characteristic of the anion. ${ }^{6}$ Finally, in lowtemperature but not rigid media, organic donors were found to sensitize the characteristic phosphorescent emission of $\mathrm{Cr}(\mathrm{NCS})_{0}{ }^{3+}{ }^{7}$

The above are all spectroscopic situations; we were interested in whether excitation energy transfer could lead to chemical reaction. This was partly to establish the occurrence of such a process and partly as a potentially valuable approach to the study of excited state photochemistry of coordination compounds. At the time this work was initiated, it was felt that a search for intramolecular excitation energy transfer would be more likely to be successful than one for intermolecular transfer. Recently, however, sensitized photoredox decompositions of cobalt(III) ammines have been found to be facile. ${ }^{8}$

The complex selected for study was trans-4-stilbene carboxylatopentaamminecobalt(III), to be referred to as species I.

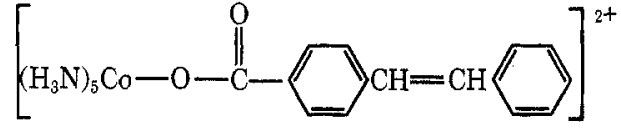

Although not a known compound, the preparation of I was not anticipated to be difficult, and the choice offered several advantages. The photochemistry of stilbene and of many of its derivatives had been studied extensively (see references 9 and 10); excitation in the region of the first singlet-singlet absorption band leads to isomerization via intersystem crossing to the first triplet excited state. Likewise, both the photochemistry and the thermal reaction chemistry of aqueous cobalt(III) ammine complexes of the type $\mathrm{Co}\left(\mathrm{NH}_{3}\right)_{5} \mathrm{X}^{2+}$ were well known (see ref 11 and 12). The first two ligand field bands of such complexes correspond to the

(1) The University, Newcastle upon Tyne, England.

(2) G. S. Hammond and R. P. Foss, J. Phys. Chem., 68, 3739 (1964).

(3) L. Lindquist, Acta Chem. Scand., 20, 2067 (1966).

(4) A. J. Fry, R. S. H. Liu, and G. S. Hammond, J. Amer. Chem. Soc., 88,4781 (1966).

(5) G. A. Crosby, R. E. Whan, and R. M. Alire, J. Chem. Phys., 34, 743 (1961).

(6) H. Gausmann and H. L. Schläfer, ibid., 48, 4056 (1968).

(7) D. J. Binet, E. L. Goldberg, and L. S. Forster, J. Phys. Chem., 72 , 3017 (1968)

(8) A. Vogler and A. W. Adamson, J. Amer. Chem. Soc., 90, 5943 (1968).

(9) J. G. Calvert and J. N. Pitts, Jr, "Photochemistry," John Wiley and Sons, Inc., New York, N. Y., 1966.

(10) G. S. Hammond, J. Saltiel, A. A. Lamola, N. J. Turro, J. S. Bradshaw, D. O. Cowan, R. C. Counsell, V. Vogt, and C. Dalton, J. Amer Chem. Soo., 86, 3197 (1964).

(11) F. Basolo and R. G. Pearson, "Mechanisms of Inorganic Reactions," 2nd ed, John Wiley and Sons, Inc., New York, N. Y., 1967.

(12) A. W. Adamson, W. L. Waltz, E. Zinato, D. W. Watts, P. D. Fleischauer, and R. D. Lindholm, Chem. Rev., 68, 541 (1968). 
transitions ${ }^{1} \mathrm{~A}_{1 \mathrm{~g}} \rightarrow{ }^{1} \mathrm{~T}_{1 \mathrm{~g}}$ and ${ }^{1} \mathrm{~A}_{1 \mathrm{~g}} \rightarrow{ }^{1} \mathrm{~T}_{2 \mathrm{~g}}$ in $\mathrm{O}_{\mathrm{h}}$ symmetry, and will be called here the $L_{1}$ and $L_{2}$ bands, respectively. If $\mathrm{X}$ is not an easily oxidizable ligand, i.e., if the energy is large for the reaction

$$
\mathrm{X}^{-}=\mathrm{X}+\mathrm{e}^{-}
$$

then the $\mathrm{L}_{1}$ and $\mathrm{L}_{2}$ bands are of normal intensity for $\mathrm{d}-\mathrm{d}$ transitions (extinction coefficients of about 70 $M^{-1} \mathrm{~cm}^{-1}$ at the band maxima), and the complexes are only slightly photosensitive in this wavelength region. Thus for $\mathrm{X}=\mathrm{Cl}$ or $\mathrm{SO}_{4}$, photolysis quantum yields are around $10^{-3}$ in the $370-\mathrm{m} \mu$ and longer wavelength region. ${ }^{13}$ The same is true for $\mathrm{Co}\left(\mathrm{NH}_{3}\right)_{6}{ }^{3+8,13}$ and $\mathrm{Co}\left(\mathrm{NH}_{3}\right)_{5}\left(\mathrm{H}_{2} \mathrm{O}\right)^{3+}, 8$ and would be expected to be true if $X$ were a carboxylato ligand, $X=R C O O$. Thus it was anticipated that irradiation of I could be made in the visible and near-ultraviolet without there being any appreciable complication due to direct photolysis of the complex.

The general expectation was that either irradiation of the $L_{1}$ band of complex I might lead to trans to cis isomerization of the stilbene moiety, or that irradiation of the singlet-singlet band of the ligand might result in either aquation or in redox decomposition of the complex. It will be seen that the latter process occurs, but apparently not the former.

\section{Experimental Section}

Preparations. The sodium salt of trans-4-stilbenecarboxylate, NaTSC, was prepared from $\alpha$-bromo- $p$ toluic acid by a literature procedure ${ }^{14}$ the latter similarly having been prepared from $p$-toluic acid. ${ }^{15}$ The corresponding acid, HTSC, was also obtained; its melting point was $239-246^{\circ}$, compared to the reported value of $247-249^{\circ} .{ }^{16}$ While the HTSC was evidently not entirely pure, the pmr spectrum was closely similar to that for trans stilbene itself, ${ }^{17}$ and no resonances attributable to the cis isomer were observed. The visible and ultraviolet spectrum of the HTSC agreed within experimental error with the published one. ${ }^{16}$

The perchlorate salt of I, $\left[\mathrm{Co}\left(\mathrm{NH}_{3}\right)_{5}(\mathrm{TSC})\right]-$ $\left(\mathrm{ClO}_{4}\right)_{2}$, was prepared as follows. Equimolar amounts of $\left[\mathrm{Co}\left(\mathrm{NH}_{3}\right)_{5}\left(\mathrm{H}_{2} \mathrm{O}\right)\right]\left(\mathrm{ClO}_{4}\right)_{3}$ and NaTSC were dissolved in a minimum volume of dimethylacetamide and the mixture was heated on a water bath at $80^{\circ}$ for $3 \mathrm{hr}$. The reaction mixture was shaken periodically; light was excluded. The crude product was obtained by pouring the reaction mixture into three to four volumes of dilute perchloric acid and then filtering off the precipitated complex. The product was recrystallized from hot $\left(90^{\circ}\right)$ water, using added sodium perchlorate to reduce the solubility. The solution was then filtered, the precipitate washed twice with cold water, once with a small volume of ethanol, and several times with ether; it was then vacuum dried over magnesium perchlorate (in the dark). The whole recrystallization procedure

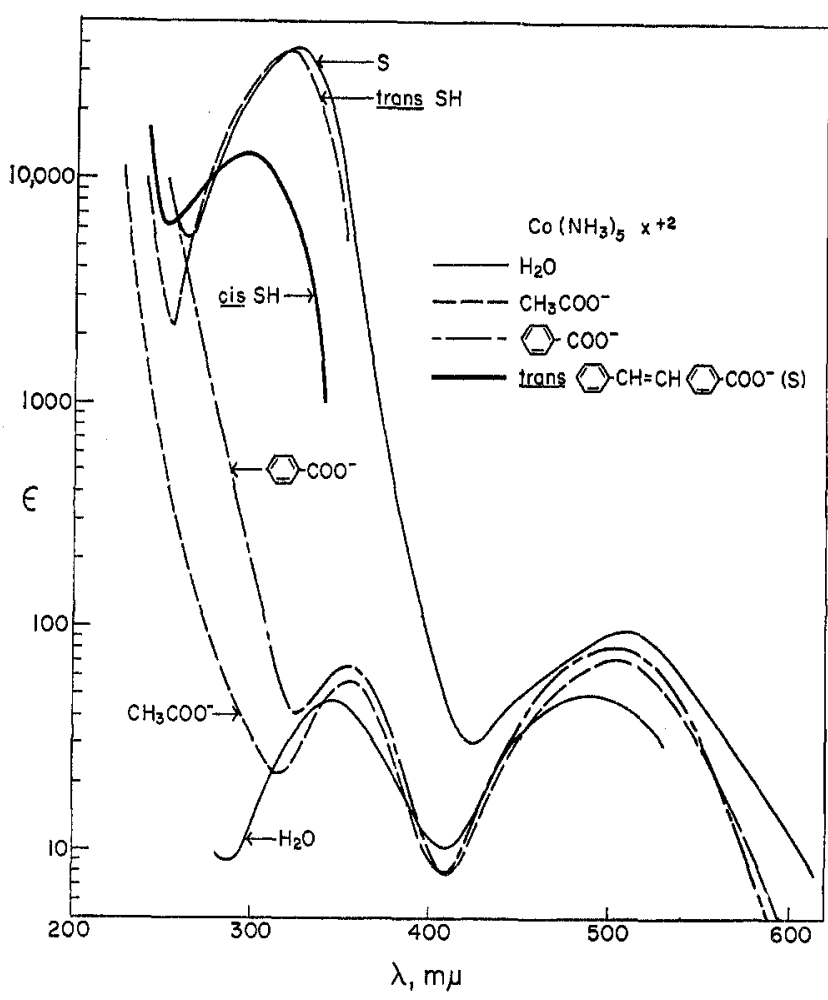

Figure 1. Absorption spectra for aqueous complexes of the type $\mathrm{Co}\left(\mathrm{NH}_{3}\right)_{5} \mathrm{X}^{2+}$ (after A. W. Adamson, Coordin. Chem. Rev., 3, 169 (1968)).

was repeated to remove traces of uncomplexed HTSC. Anal. Calcd for $\left[\mathrm{Co}\left(\mathrm{NH}_{3}\right)_{5}\left(\mathrm{O}_{2} \mathrm{C}_{15} \mathrm{H}_{11}\right)\right]\left(\mathrm{ClO}_{4}\right)_{2}$ : C, $31.5 ; \mathrm{H}, 5.1 ; \mathrm{N}, 12.3$. Found: $\mathrm{C}, 31.8 ; \mathrm{H}, 4.6$; $\mathrm{N}, 12.4$. The absorption spectrum of the aqueous solution of $I$ is included in Figure 1.

The cis isomer of 4-stilbenecarboxylic acid, HCSC, was prepared by irradiating a dioxane solution of HTSC for several hours with a mercury arc lamp. The product was isolated by the procedure of ref 16 .

Benzoatopentaamminecobalt(III) perchlorate was prepared by the method of Gould and Taube, ${ }^{18}$ and recrystallized twice from water. Anal. Calcd for $\left[\mathrm{Co}\left(\mathrm{NH}_{3}\right)_{5}\left(\mathrm{O}_{2} \mathrm{C}-\mathrm{C}_{6} \mathrm{H}_{5}\right)\right]\left(\mathrm{ClO}_{4}\right)_{2} \cdot 2 \mathrm{H}_{2} \mathrm{O}: \quad \mathrm{C}, \quad 16.80 ; \mathrm{H}$, 4.80 ; N, 14.00; Co, 11.80. Found: C, $17.06 \mathrm{H}, 4.77$; $\mathrm{N}, 13.86 ; \mathrm{Co}, 12.10$. A sample of pure acetatopentaamminecobalt(III) perchlorate was kindly supplied by Dr. M. Barret. The spectra of these last two complexes are included in Figure 1.

General Procedures. The light source was a watercooled General Electric A-H6 mercury arc, mounted

(13) A. W. Adamson, Discussions Faraday Soc., 29, 163 (1960).

(14) D. H. Wadsworth, O. E. Schupp, E. J. Sens, and J. A. Ford, Jr., J. Org. Chem., 30, 680 (1965).

(15) W. Davies and W. H. Perkins, J. Chem. Soc., 2202 (1922).

(16) G. Berti and F. Bottari, Gazz. Chim. Ital., 89., 2371 (1959).

(17) "Catalogue of NMR Spectra," Varian Associates, Palo Alto, Calif., spectra no. 305 and 306 .

(18) E. S. Gould and H. Taube, J. Amer. Chem. Soc., 86, 1318 (1964). 
on an optical bench, with collimating lenses, etc., as previously described. ${ }^{19}$ A UG11 Schott glass filter was used for the irradiations of the stilbene absorption band; this filter transmitted light between 250 and 385 $\mathbf{m} \mu$, but since Pyrex optics were used, the net wavelength spread of the light reaching the solution was limited to the range 340 to $385 \mathrm{~m} \mu$. Two Corning glass filters, CS-370 and CS-496, were used in combination for irradiation of the $\mathrm{L}_{1}$ bands of the complexes; this combination transmitted light between 500 and $600 \mathrm{~m} \mu$.

The photolyses were carried out in a 10-cm cylindrical spectrophotometer cell, using solutions of such known concentration (around $0.001 \mathrm{M}$ ) as to have complete absorption of the incident light. To avoid $\mathrm{pH}$ increases during photolyses, which might lead to base catalyzed hydrolysis of the complexes, the solutions were also $0.01 M$ in perchloric acid. In addition, the photolyses were limited to about $10 \%$ reaction, to avoid undue inner filter effects and, in the case of solutions of $I$, to minimize loss of light from scattering by the insoluble HTSC produced. Incident light intensity was determined by Reineckate actinometry. ${ }^{20}$

Emission spectra were measured by means of an American Instrument Company spectrofluorometer, and the visible and ultraviolet absorption spectra by means of a Cary Model 14 spectrophotometer or a Beckman Model DU instrument. Proton resonance spectra were obtained with a Varian A60 instrument.

Analytical Procedures. Carbon, hydrogen, and nitrogen analyses were made commercially by standard microanalytical procedures (Elek Microanalytical Laboratories, Torrance, Calif.).

The photolyzed solutions were analyzed for the various possible products as follows. Cobalt(II) was determined by dilution of an aliquot of the solution into acetone containing ammonium thiocyanate, and the optical density due to $\mathrm{Co}(\mathrm{NCS})_{4}{ }^{2-}$ measured at 625 $\mathrm{m} \mu{ }^{21}$ Free HTSC released during the photolyses was present as a suspension since the acid is quite insoluble in acidified water. It was extracted with ether, the ether solution evaporated, and the residue triturated with $2 \quad M$ sodium hydroxide and the mixture filtered. The remaining precipitate consisted of $\mathrm{Na}$ TSC, which is insoluble under these conditions. The solid was converted to HTSC by the addition of dilute aqueous hydrochloric acid and dissolved in ethanol; its identity was confirmed by its absorption spectrum, and the amount present by the absorbance at 319 $\mathrm{m} \mu .^{16}$

The filtrate from the above separation contained any NaCSC present, as established by tests with a sample of known material. Where such product was present, it was then precipitated as the acid, filtered off, dissolved in ethanol, and determined spectrophotometrically.
A further possible product was $\mathrm{Co}\left(\mathrm{NH}_{3}\right)_{5}\left(\mathrm{H}_{2} \mathrm{O}\right)^{3+}$. The photolyzed solution was passed through a Dowex 50 ion-exchange column, and any cobaltous ion and aquopentaammine complex present was then eluted with $10 \%$ aqueous hydrochloric acid. The aquo complex was then determined from the visible and ultraviolet absorption spectrum of the effluent solution.

\section{Results}

The principal results are summarized in Table I. Both the acetato- and the benzoatopentaammine complexes showed the expected low sensitivity to light in the region of either the $L_{1}$ or the $L_{2}$ absorption bands. A small yield of the aquation product, $\mathrm{Co}\left(\mathrm{NH}_{3}\right)_{5^{-}}$ $\left(\mathrm{H}_{2} \mathrm{O}\right)^{3+}$, appeared to be present, but insufficient for an accurate determination. An upper limit to the amount could be set, however, which was adequate for the purposes of this investigation.

Table I: Photolysis of Certain Carboxylatopentaamminecobalt(III) Complexes $\left(25^{\circ}, 0.01 M\right.$ Aqueous Perchloric Acid)

\begin{tabular}{|c|c|c|}
\hline \multirow[b]{2}{*}{ Complex } & \multicolumn{2}{|c|}{ Quantum yields ${ }^{a}$} \\
\hline & $340-385 \mathrm{~m} \mu$ & $500-600 \mathrm{~m} \mu$ \\
\hline $\mathrm{Co}\left(\mathrm{NH}_{3}\right)_{5}(\text { acetate })^{2+}$ & $\phi_{R}<7 \times 10^{-4}$ & $\begin{array}{l}\phi_{\mathrm{R}}<1 \times 10^{-4} \\
\phi_{\mathrm{A}}<0.01\end{array}$ \\
\hline $\mathrm{Co}\left(\mathrm{NH}_{3}\right)_{5}$ (benzoate $)^{2+}$ & $\begin{array}{l}\phi_{\mathrm{R}}<3 \times 10^{-3} \\
\phi_{\mathrm{A}}<0.01\end{array}$ & $\phi_{A}<0.01$ \\
\hline $\mathrm{Co}\left(\mathrm{NH}_{3}\right)_{5}(\mathrm{TSC})^{2+}$ & $\begin{aligned} & \phi_{\mathrm{R}}=0.16 \\
& \phi_{\mathrm{L}}=0.090 \\
& \phi_{\mathrm{A}}<0.04 \\
& b\end{aligned}$ & $\begin{aligned} \phi_{\mathrm{R}} & <1 \times 10^{-4} \\
\phi_{\mathrm{A}} & <0.05\end{aligned}$ \\
\hline
\end{tabular}

${ }^{a} \phi_{R}, \phi_{A}$, and $\phi_{L}$ denote the quantum yields for $\mathrm{Co}$ (II), aquopentaamminecobalt(III), and free TSC ligand, respectively. ${ }^{b}$ The quantum yield for isomerization of the released TSC was less than $1 \times 10^{-3}$, and less than $5 \times 10^{-4}$ for that which remained coordinated.

By contrast, complex I was quite light sensitive in the region $340 \mathrm{~m} \mu$ to $385 \mathrm{~m} \mu$. The principal reaction was one of redox decomposition to give cobalt(II); the actual oxidation products were not determined, but the TSC ligand was affected in part since the yield of free HTSC was only about half of that of cobalt(II). Again, it was established that only a small proportion of the photolysis led to formation of $\mathrm{Co}\left(\mathrm{NH}_{3}\right)_{5}\left(\mathrm{H}_{2} \mathrm{O}\right)^{3+}$ and also therefore that this reaction mode was not responsible for any important fraction of the free HTSC found. Ammonia analyses were not accurate enough to determine whether some $\mathrm{Co}\left(\mathrm{NH}_{3}\right)_{4}\left(\mathrm{H}_{2} \mathrm{O}\right)$ (TSC) ${ }^{2+}$ might have been formed, analogous to the reported behavior of $\mathrm{Co}\left(\mathrm{NH}_{3}\right)_{5} \mathrm{Cl}^{2+22}$ and $\mathrm{Co}\left(\mathrm{NH}_{3}\right)_{5}-$

(19) E. Zinato, R. Lindholm, and A. W. Adamson, J, Amer, Chem. Soc., 91,1076 (1969).

(20) E. Wegner and A. W. Adamson, ibid., 88, 394. (1966),

(21) R. E. Kitson, Anal. Chem., 22, 664 (1959).

(22) L. Moggi, N. Sabbatini, and V. Balzani, Gazz. Chim. Ital., 97, 980 (1967). 
$\mathrm{N}_{3}{ }^{2+23}$ (in these last two cases, however, $254 \mathrm{~m} \mu$ radiation was used, so that a charge-transfer band was irradiated).

It was established that no isomerization of the TSC occurred. The free TSC ligand contained no appreciable amount of cis form. The second possibility, namely that ligand isomerization occurred but with the TSC remaining coordinated, was ruled out by photolyzing a solution of $\mathrm{I}$ to complete decomposition. Again, no cis ligand was found (the free HTSC produced in the irradiation remained suspended as insoluble material, and hence was not subject to any appreciable photoisomerization itself).

As indicated in Table I, complex I was virtually insensitive to light around $500 \mathrm{~m} \mu$ in wavelength, as was the acetatopentaammine species. Finally, it should be mentioned that the dark thermal aquation of the various complexes was small enough that no correction for this was needed. Also, in the case of I, deaeration of the solution did not affect the photochemical behavior.

The results of the emission studies, using $330 \mathrm{~m} \mu$ stimulating light, are shown in Figure 2. The free ligand, as NaTSC, showed the expected fluorescent emission centered at $400 \mathrm{~m} \mu$, characteristic of the first excited singlet state. ${ }^{24}$ No phosphorescent emission was observed, nor expected, in terms of the behavior of stilbenes generally in room temperature solution. ${ }^{25}$

As shown in the figure, quite appreciable quenching of the fluorescence occurred in the presence of added $\mathrm{Co}\left(\mathrm{NH}_{3}\right)_{5}\left(\mathrm{H}_{2} \mathrm{O}\right)^{3+}$, and emission was essentially absent in the case of compound I. The small residual emission shown in Figure 2 for this last case is almost certainly due to the presence of some free TSC produced by photodecomposition. The results are for a $50 \%$ water-ethanol mixture as solvent; this medium was chosen as one in which all of the species had a reasonable solubility and yet would still be largely aqueous.

\section{Discussion}

The immediate conclusion from the above results seems to be that intramolecular energy transfer occurs with $I$, to provide an example of an internally sensitized photolysis of a coordination compound. The basis for this conclusion follows.

The fact that the absorption band of I at $320 \mathrm{~m} \mu$ is essentially unaltered from that for free HTSC indicates that the singlet-singlet transition has remained largely and perhaps entirely isolated in the ligand moiety. Alternatively, it does not seem tenable to view the 320$\mathrm{m} \mu$ band in I as a charge-transfer band of the type found in acidopentaammine complexes (CTTM), not only for the above reason, but also because the other carboxylatopentaammines show a normal intensity $\mathrm{L}_{2}$ absorption in that wavelength region. It could be argued that the extended $\pi$ system of the TSC ligand

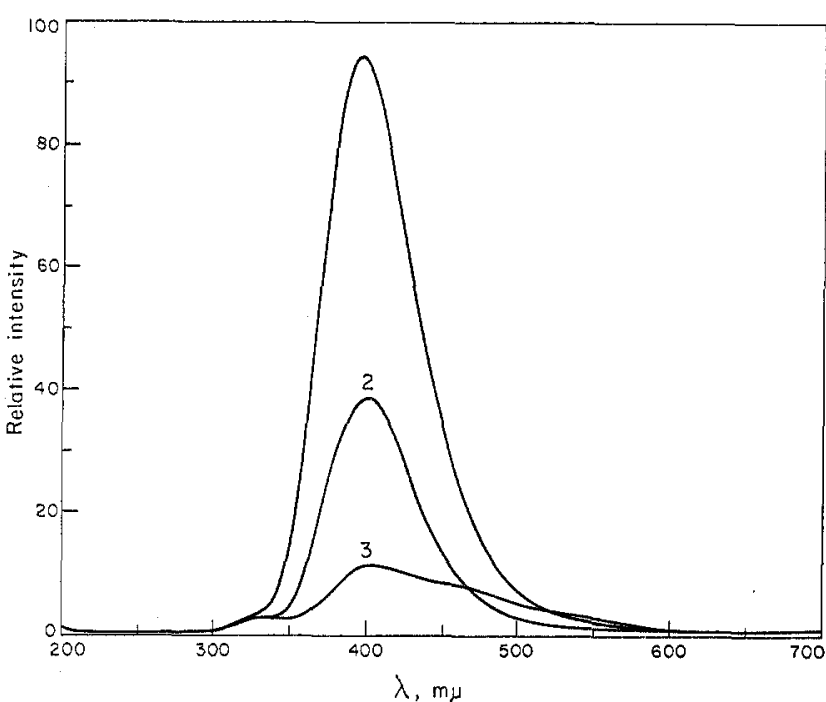

Figure 2. Emission from trans-4-stilbenecarboxylate ion (330 $\mathrm{m} \mu$ exciting radiation; $50 \%$ ethanol-water solution): $1,0.001$ $M$ NaTSC; $2,0.001 M$ NaTSC $+0.005 M \mathrm{Co}\left(\mathrm{NH}_{3}\right)_{5}\left(\mathrm{H}_{2} \mathrm{O}\right)^{3+}$ 3, $0.001 M$ complex I.

might shift the first CTTM band of I to the $370-\mathrm{m} \mu$ region, and it is true that some bathochromic shift appears to occur in the case of the benzoatepentaammine complex, relative to the acetatopentaammine one. However, the virtually intact minimum in the absorption spectrum of I at about $250 \mathrm{~m} \mu$ should not then be present. From a comparison of the minima for I and for free TSC, a maximum of a few per cent contribution from a hidden CTTM band might be present, or not enough for absorption by such a hidden band to account for the observed quantum yield of 0.16 .

As confirmation that the carboxyl group as a ligand does not introduce appreciable CTTM character to the $\mathrm{L}_{2}$ band, the acetato- and benzoatopentaammine complexes show about the same low photosensitivity in the 340 to $385-\mathrm{m} \mu$ region as do the hexaammine and aquopentaammine species.

It seems clear, then, that the redox photolysis that occurs with I should not be ascribed to any disposition toward such reaction of the excited state first reached by absorption in 320-m $\mu$ band. As further discussed below, the fact that the observed reaction is one of redox decomposition strongly suggests that the excited state of the coordinated cobalt system which is populated by energy transfer is one having CTTM character.

To elaborate on the above conclusion, the partial quenching of the fluorescent emission from TSC by $\mathrm{Co}\left(\mathrm{NH}_{8}\right)_{5}\left(\mathrm{H}_{2} \mathrm{O}\right)^{3+}$ and the essentially complete quenching when TSC is coordinated in I probably represent

(23) J. F. Endicott and M. Z, Hoffman, J. Amer. Chem. Soc., 90, 4740 (1968).

(24) H. Dyck and D. S. McClure, J. Chem. Phys., 36, 2326 (1962). (25) D. Schulte-Frohlinde, H. Blume, and H. Gusten, J. Phys. Chem., $66,2486(1962)$. 
analogous processes. In the first case, the quenching is accompanied by redox decomposition of the aquopentaammine complex, ${ }^{8}$ and in the second, by the redox decomposition of I. Both effects could be supposed to be due to a catalyzed deactivation of the singlet excited state of the TSC group, but then the associated photolysis would have to be attributed to a hot ground state reaction of the complex. Were this the case, aquation would be the expected reaction as this is an easier thermal process than is redox decomposition. As with acidopentaammines of cobalt(III) generally, the carboxylatopentaammines show a slow thermal aquation of the acido group, but no detectable redox decomposition.

It seems necessary, then, to ascribe the quenching to an excitation energy transfer rather than to deactivation catalysis. A second supposition would be that the produced excited state was a cobalt(III) ligand field state, i.e., one derived from the ${ }^{1} \mathrm{~T}_{1 \mathrm{~g}}$ or ${ }^{1} \mathrm{~T}_{2 \mathrm{~g}}$ states of $\mathrm{O}_{\mathrm{h}}$ symmetry. This supposition can also be ruled out on the grounds that the observed photochemistry is inappropriate to it. Thus direct population of such excited states by absorption in the region of the $L_{1}$ or $L_{2}$ bands of the acetato- and benzoatopentaammine complexes, and of the $L_{1}$ band of complex (I) leads only to very low quantum yield processes, probably mainly aquation in nature. Such population by energy transfer should do likewise, contrary to the high photoredox yield found for $I$.

It thus seems necessary to conclude not only that excitation energy transfer has occurred, but also that fer. The second alternative was considered to be the more likely in the case of the biacetyl sensitized decomposition of $\mathrm{Co}\left(\mathrm{NH}_{3}\right)_{6}{ }^{3+},{ }^{8}$ since in the presence of this complex, the biacetyl phosphorescence was quenohed, but not the fluorescence. In the case of TSC, however, fluorescence quenching is observed, which indicates that the singlet excited state has been intercepted.

A further point is that in the ordinary photochemistry of the stilbenes, the observed trans to cis photoisomerization is considered to occur via the triplet excited state (see ref 9 and 10), produced from the singlet excited state by intersystem crossing. In our system we observe no isomerization either of the TSC which remains coordinated or of that which is released, which suggests that the triplet excited state of TSC was not populated. We therefore conclude that most probably the triplet CTTM state of the complex is formed from the singlet excited state of the TSC ligand by an intramolecular crossing process. The spin conservation rule is not of ubiquitous importance; it is violated, for example, in singlet to triplet intersystem crossing processes.

A remaining speculation concerns the chemical mechanism of the redox decomposition of I. It follows from the quantum yield results that production of cobalt(II) leads to free TSC ligand only about 55\% of the time. The implication is that $55 \%$ of the primary acts lead to oxidation either of ammonia or of solvent, and $45 \%$, to oxidation of the TSC. As illustrated by the mechanistic scheme below, the primary

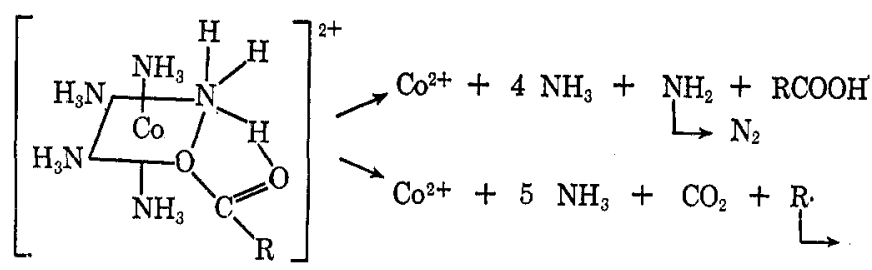

produced state is a CTTM state of the cobalt(III)ligand system. The spectroscopically observed first CTTM band for the carboxylatopentaammine complexes lies around $200 \mathrm{~m} \mu$, however, and it does not seem possible to populate this state through the singlet excited state of the TSC group, on energy grounds. As previously noted, ${ }^{8}$ though, there must exist a set of triplet CTTM excited states of cobalt(III) complexes which are nonspectroscopic (i.e., are not seen in absorption spectroscopy), but which might be reached by intersystem crossing processes. It appears to us that in the present system as well as in those involving intermolecular sensitization, it is a low-lying, nonspectroscopic triplet CTTM state which is responsible for the observed photoredox reaction.

A remaining question is whether the above state is produced directly from the TSC singlet excited state or whether the latter first converts to a TSC triplet state which then undergoes the excitation energy trans- process might be reaction 2 part of the time. This is apparently what occurs in the $254-\mathrm{m} \mu$ photolysis of $\mathrm{Co}\left(\mathrm{NH}_{3}\right)_{5}\left(\mathrm{H}_{2} \mathrm{O}\right)^{3+}$, for which the final products are cobalt(II) and nitrogen. ${ }^{26}$ Hydrogen bonding between the carboxyl oxygen and an adjacent ammonia group is likely, and a reaction of type 2 might be assisted by a hydrogen atom transfer to the TSC ligand. Reaction 3 shows possible products if the acido group is oxidized directly. The ultimate fate of possible fragments such as $R$. was not sought for in the present investigation.

Acknowledgment. These investigations were supported in part by Contract No. DA-31-124-ARO-D-343 between the University of Southern California and the U. S. Army Research Office (Durham).

(26) J. F. Endicott and M. Z. Hoffman, J. Amer. Chem. Soc., 87, 3348 (1965) 